\title{
Analysis of Glipizide Binding to Normal and Glycated Human Serum Albumin by High-Performance Affinity Chromatography
}

\author{
Ryan Matsuda, Zhao Li, Xiwei Zheng, and David S. Hage ${ }^{*}$ \\ Department of Chemistry, University of Nebraska-Lincoln, Lincoln, NE 68588-0304 (USA)
}

\begin{abstract}
In diabetes, the elevated levels of glucose in the blood stream can result in the non-enzymatic glycation of proteins such as human serum albumin (HSA). This type of modification has been shown to affect the interactions of some drugs with HSA, including several sulfonylurea drugs that are used to treat type II diabetes. This study used high-performance affinity chromatography (HPAC) to examine the interactions of glipizide (i.e., a second-generation sulfonylurea drug) with normal HSA or HSA that contained various levels of in vitro glycation. Frontal analysis indicated that glipizide was interacting with both normal and glycated HSA through two general groups of sites: a set of relatively strong interactions and a set of weaker interactions with average association equilibrium constants at $\mathrm{pH} 7.4$ and $37^{\circ} \mathrm{C}$ in the range of $2.4-6.0 \times 10^{5} \mathrm{M}^{-1}$ and 1.7$3.7 \times 10^{4} \mathrm{M}^{-1}$, respectively. Zonal elution competition studies revealed that glipizide was interacting at both Sudlow sites I and II, which were estimated to have affinities of 3.2-3.9 $\times 10^{5}$ $\mathrm{M}^{-1}$ and 1.1-1.4 $\times 10^{4} \mathrm{M}^{-1}$. Allosteric effects were also noted to occur for this drug between the tamoxifen site and the binding of $R$-warfarin at Sudlow site I. Up to an $18 \%$ decrease in the affinity for glipizide was observed at Sudlow site I in going from normal HSA to glycated HSA, while up to a $27 \%$ increase was noted at Sudlow site II. This information should be useful in indicating how HPAC can be used to investigate other drugs that have complex interactions with proteins. These data should also be valuable in providing a better understanding of how glycation may affect drug-protein interactions and the serum transport of drugs such as glipizide during diabetes.
\end{abstract}

\section{Keywords}

Glipizide; Human serum albumin; Glycation; High-performance affinity chromatography; Drugprotein binding

\section{Introduction}

An estimated 366 million people in the world and 25.8 million people in the United States are affected by diabetes [1,2]. Diabetes is a disease that is associated with elevated levels of glucose in the blood stream, which can result in non-enzymatic protein glycation [3-9]. Glycation involves the reaction between glucose and free amine groups on proteins [3-9].

*Author for correspondence: Chemistry Department, University of Nebraska, Lincoln, NE 68588-0304 USA. Phone: 402-472-2744; FAX: 402-472-9402; dhage1@unl.edu. 
This process initially results in the formation of a Schiff base, which can undergo further rearrangement to form a stable Amadori product. Glycation has been a topic of growing interest due to its possible effect on proteins and tissues $[9,10]$. One protein that has been examined in recent years in such work is human serum albumin (HSA), with diabetic patients having a 2- to 5-fold increase in the amount of glycated HSA versus persons who do not have diabetes [11].

HSA is the most abundant serum protein $[12,13]$. This protein has a single chain of 585 amino acids and a molecular weight of $66.5 \mathrm{kDa}[12,13]$. HSA is involved in several physiological processes, including the transportation of low mass substances such as hormones, fatty acids, and drugs [5,12,14-17]. There are two major binding sites for drugs on HSA: Sudlow sites I and II $[12,18,19]$. Drugs that bind to Sudlow site I include bulky heterocyclic anionic drugs such as warfarin, azapropazone, phenylbutazone, and salicylate $[12,14,18,20]$, while drugs or solutes such as ibuprofen, fenoprofen, ketoprofen, benzodiazepine, and L-tryptophan bind to Sudlow site II [12,14,18,21]. There are also other regions on HSA that can bind to fatty acids and to drugs like tamoxifen or digitoxin [1417,22-24].

Sulfonylurea drugs are orally-administered drugs that are commonly used to treat type II diabetes $[2,25]$. The core structure of a sulfonylurea drug is shown in Fig. 1. Sulfonylurea drugs are known to bind tightly to serum proteins, and especially to HSA [10]. Several studies have recently examined the binding of sulfonylurea drugs to HSA and glycated forms of this protein [26-34]. Some common first-generation sulfonylurea drugs (e.g., tolbutamide and acetohexamide) and a number of second-generation drugs (e.g., gliclazide and glibenclamide) have all been found to bind at both Sudlow sites I and II on normal HSA and glycated HSA [26-31]. Glibenclamide has also been found to interact with the digitoxin site of HSA [31].

The purpose of this report is to examine binding by glipizide to normal HSA and HSA at various stages of in vitro glycation. Glipizide (see Fig. 1) is a second-generation sulfonylurea drug that has a limited solubility in water (approximately $20 \mathrm{mg} / \mathrm{L}$ ) and is relatively lipophilic $(\log P, 2.31)$. These properties have made it difficult to work with this drug under physiological conditions and when using some traditional methods for binding studies (e.g., fluorescence spectroscopy) [34-38]. An alternative approach that will be used in this report for such studies is high-performance affinity chromatography (HPAC).

HPAC is a type of HPLC that uses an immobilized biologically-related agent (e.g., HSA) as a stationary phase to retain specific analytes [40,41]. HPAC has frequently been used for the isolation and analysis of specific targets; however, this technique can also be employed for characterizing biological interactions [40-42]. This has included the recent use of HPAC in examining the effects of glycation on the binding of several first- and second-generation sulfonylurea drugs to HSA [26-33]. Advantages of HPAC for this work are its ability to determine both the strength of a drug-protein interaction and the number or location of these binding sites [40,41]. Recent studies have also demonstrated that HPAC can be used with drugs that have limited solubility in aqueous buffers [31]. 
This report will use HPAC to examine both the overall binding and site-specific interactions of glipizide with HSA, based on frontal analysis and zonal elution competition studies. These experiments will be conducted with both normal HSA and HSA that has been prepared in vitro to have glycation levels representative of pre-diabetes or diabetes. HPAC will be used to identify the major binding sites for glipizide on HSA and to characterize the affinities of these sites for this drug, or the interactions between such sites (e.g., positive or negative allosteric effects). These experiments should provide a better understanding of how HPAC can be used to examine binding by drugs that may have relatively complex interactions with a protein. The results should also help to build a more complete picture of how non-enzymatic glycation may alter the binding of drugs such as glipizide to HSA and affect the serum protein binding and transport of such a drug during diabetes.

\section{Experimental}

\section{Chemicals}

The glipizide ( $\geq 96 \%$ pure), $R$-warfarin ( $\geq 97 \%$ ), racemic warfarin ( $\geq 99 \%$ ), L-tryptophan ( $\geq$ $97 \%$ ), digitoxin ( $\geq 97 \%$ ), tamoxifen ( $\geq 99 \%)$, $\beta$-cyclodextrin (>98\%), D-(+)-glucose ( $\geq$ $99.5 \%$ ), sodium azide $(95 \%)$, and HSA (essentially fatty acid free, $(\geq 96 \%)$ were purchased from Sigma Aldrich (St. Louis, MO, USA). Nucleosil Si-300 (7 $\mu$ m particle diameter, $300 \AA$ pore size) was obtained from Macherey-Nagel (Düren, Germany). A fructosamine assay kit was purchased from Diazyme Laboratories (San Diego, CA, USA) for measuring the modification levels of the glycated HSA samples. Reagents for the bicinchoninic acid (BCA) protein assay were from Pierce (Rockford, IL, USA). A Milli-Q-Advantage A 10 system (EMD Millipore Corporation, Billerica, MA, USA) was used to purify the water utilized to make all aqueous solutions, which were also filtered through $0.20 \mu \mathrm{m}$ GNWP nylon membranes from Millipore.

\section{Instrumentation}

The chromatographic system was from Jasco (Tokyo, Japan) and consisted of two PU-2080 pumps, a DG-2080 degasser, an AS-2057 autosampler, a CO-2060 column oven, and an UV-2075 absorbance detector, plus a Rheodyne Advantage PF six-port valve (Cotati, CA, USA). The system was controlled by Jasco LCNet through use of ChromNav software. PeakFit 4.12 (Jandel Scientific Software, San Rafael, CA, USA) was used to analyze the chromatograms. Non-linear regression analysis was carried out by using Data Fit 8.1.69 (Oakdale, PA, USA).

\section{In-vitro Glycation of HSA}

Two samples of glycated HSA (referred to here as gHSA1 and gHSA2) were prepared in vitro at a physiological concentration and $\mathrm{pH}$, according to previous methods [29,43,44]. All materials used for this procedure (e.g., glassware and spatulas) were first sterilized through autoclaving to prevent bacterial growth during the glycation process. A sterile $\mathrm{pH} 7.4,0.20$ $\mathrm{M}$ potassium phosphate buffer was also prepared in this manner. Further prevention of bacterial growth was achieved through the addition of $1 \mathrm{mM}$ sodium azide to this buffer. 
Normal HSA (i.e., $840 \mathrm{mg}$ ) was placed into $20 \mathrm{~mL}$ of the $\mathrm{pH} 7.4$ buffer (giving a final concentration of $42 \mathrm{mg} / \mathrm{L} \mathrm{HSA}$ ) along with a moderate or high concentration of D-glucose (i.e., 15 or $30 \mathrm{mM}$ ). These mixtures were incubated for four weeks at $37^{\circ} \mathrm{C}$. The samples were then lyophilized and stored at $-80^{\circ} \mathrm{C}$. A fructosamine assay [29] was performed in duplicate to determine the glycation level of the HSA samples, given values for the normal HSA, gHSA1 and gHSA2 of $0.24( \pm 0.13), 1.39( \pm 0.28)$ and $3.20( \pm 0.13) \mathrm{mol}$ hexose/mol HSA, respectively.

\section{Preparation of Supports and Columns}

The chromatographic supports were prepared by first converting Nucleosil Si-300 silica into a diol-bonded form and then immobilizing HSA or glycated HSA to this material through the Schiff base method [45-48]. Although free amine groups are involved in both the Schiff base immobilization method and glycation, these processes tend to involve different residues on HSA, making the resulting protein support a good model for the soluble forms of normal HSA or glycated HSA [26-33,49]. A control support was prepared in this manner, but with no protein being added during the immobilization step. A BCA assay was performed on a dried portion of each support to determine its protein content. This assay was performed in triplicate using soluble HSA as the standard and the control support used as a blank. The measured protein content was $97( \pm 2), 85( \pm 4)$, and $95( \pm 4) \mathrm{mg} \mathrm{HSA} / \mathrm{g}$ silica for the normal HSA, gHSA1, and gHSA2 supports, respectively.

A portion of each support was downward slurry packed into a separate $2.0 \mathrm{~cm} \times 2.1 \mathrm{~mm}$ i.d. stainless steel column at $3500 \mathrm{psi}(24 \mathrm{MPa})$ and using a packing solution that was $\mathrm{pH} 7.4$, $0.67 \mathrm{M}$ potassium phosphate buffer. The packed columns and any remaining support were stored at $4{ }^{\circ} \mathrm{C}$ in $\mathrm{pH} 7.4,0.067 \mathrm{M}$ phosphate buffer until further use. These columns remained stable throughout the course of this entire study (i.e., over the course of 500 sample applications) and with no significant changes being noted in their binding properties during the described experiments, as reported in prior work with similar columns [2631,50].

\section{Chromatographic Studies}

The solutions of glipizide, $R$-warfarin, racemic warfarin, L-tryptophan, digitoxin, and tamoxifen were prepared in $\mathrm{pH} 7.4,0.067 \mathrm{M}$ phosphate buffer. Digitoxin and tamoxifen have limited solubility in aqueous solutions (i.e., around $4 \mathrm{mg} / \mathrm{L}$ and $0.17 \mathrm{mg} / \mathrm{L}$, respectively), so a solubilizing agent (i.e., $\beta$-cyclodextrin) was also placed into their solutions [51,52]. The addition of this solubilizing agent to only the injected samples has been noted in prior studies with HPAC to not cause any notable changes in the measured binding parameters for the applied drugs with an immobilized protein such as HSA $[23,51,52]$. Digitoxin was prepared in a $0.88 \mathrm{mM} \beta$-cyclodextrin solution, and tamoxifen was prepared in a $2.2 \mathrm{mM} \beta$-cyclodextrin, as described previously [22-24]. The racemic warfarin, $R$-warfarin, digitoxin, and tamoxifen solutions were prepared and used within two weeks of preparation [29,50,53]. Solutions of L-tryptophan in $\mathrm{pH}$ 7.4, $0.067 \mathrm{M}$ phosphate buffer are known to be stable for about 2-9 days, so these particular solutions were used within one day of preparation [29,53,54]. Glipizide required additional steps to place this drug into an aqueous solution [31]. To do this, a stock solution containing $50 \mu \mathrm{M}$ glipizide in 
$\mathrm{pH} 7.4,0.067 \mathrm{M}$ phosphate buffer was first made by dissolving this drug through the aid of repeated $4 \mathrm{~h}$ periods of sonication over 5 days. This stock solution was then used to prepare diluted working solutions of glipizide for the chromatographic experiments. All of the glipizide solutions were used within 2 weeks of preparation. Although sulfonylurea drugs are weak acids (e.g., the $\mathrm{p} K_{\mathrm{a}}$ of glipizide is 5.9), the drug concentrations that were used in these experiments had little or no effect on the final $\mathrm{pH}$ of the buffered solutions [34,55].

The mobile phases used for the chromatographic experiments were prepared in or diluted with $\mathrm{pH}$ 7.4, $0.067 \mathrm{M}$ phosphate buffer. The same buffer was used as the isocratic application/elution buffer during sample injection or application. All of the mobile phases were passed through a $0.2 \mu \mathrm{m}$ filter and degassed for 10-15 min prior to use. The chromatographic experiments were carried out at a typical flow rate of $0.50 \mathrm{~mL} / \mathrm{min}$ and at a temperature of $37^{\circ} \mathrm{C}$. As has been demonstrated with similar columns and systems, these experimental conditions have been shown to provide reproducible results in the measurement of drug-binding parameters such as retention factors, binding capacities and association equilibrium constants [26-31].

Prior to all chromatographic studies, the columns were first equilibrated with $\mathrm{pH}$ 7.4, 0.067 M phosphate buffer. In the frontal analysis experiments, a six-port valve was used to switch from the $\mathrm{pH} 7.4$ buffer to a solution that contained a known concentration of glipizide in this buffer. The elution of glipizide was monitored at $254 \mathrm{~nm}$. As shown in Fig. 2(a), the application of each glipizide solution resulted in the formation of a breakthrough curve. After a stable plateau had been reached in this curve, a valve was used to switch the column back to $\mathrm{pH} 7.4,0.067 \mathrm{M}$ phosphate buffer to elute the retained drug and regenerate the column.

Frontal analysis was carried out on each normal HSA or glycated HSA column and a control column by using twelve glipizide solutions with concentrations ranging from 0.5 to $50 \mu \mathrm{M}$. These conditions were all in the linear range of the detector, and the experiments at each drug concentration were carried out in quadruplicate. The breakthrough curves were processed by using the first derivative function of PeakFit 4.12 , followed by analysis of this derivative using the equal area function to determine the mean point of the curve [53]. The results obtained on the control column at each glipizide concentration were subtracted from the results for the normal HSA or glycated HSA columns to correct for the system void time and non-specific binding of the drug to the support. Non-specific binding to the support made up 50-56\% of the total binding observed when applying a $50 \mu \mathrm{M}$ solution of glipizide to the normal HSA or glycated HSA columns. This level of non-specific binding was similar to that obtained in prior research with glibenclamide, which successfully used the same approach to correct for such binding on HPAC columns [31].

Zonal elution competition studies, as illustrated in Fig. 2(b), were carried out by using $R$ warfarin and L-tryptophan as site-specific probes for Sudlow sites I and II, while digitoxin and tamoxifen were used as probes for the digitoxin and tamoxifen sites [22-24,26-31]. These competition studies were performed using eight solutions that contained 0.0 to 20.0 $\mu \mathrm{M}$ glipizide in the mobile phase. These solutions were also used to dilute and prepare $5 \mu \mathrm{M}$ samples of each probe compound, which were found to provide linear elution conditions for 
these compounds. The probe samples were applied to each column as $20 \mu \mathrm{L}$ injections and were monitored at 308, 280, 205, or $205 \mathrm{~nm}$ for $R$-warfarin, L-tryptophan, digitoxin or tamoxifen, respectively. Sodium nitrate was used as a non-retained solute and was applied at a concentration of $20 \mu \mathrm{M}$ and using an injection volume of $20 \mu \mathrm{L}$, while being monitored at $205 \mathrm{~nm}$ [39-42]. The experiments at each concentration of glipizide and with each sitespecific probe were performed in quadruplicate for the normal HSA or glycated HSA columns and control column. The central point of each peak was determined by using the equal area function of PeakFit 4.12 [53].

Similar competition studies were carried out in quadruplicate by using glipizide as the injected probe and racemic warfarin or tamoxifen as the competing agent. The mobile phases in these experiments contained one of eight concentrations for racemic warfarin that ranged from 0.0 to $20.0 \mu \mathrm{M}$ or one of eight concentrations for tamoxifen that ranged from 0.0 to $10.0 \mu \mathrm{M}$. Samples containing $5 \mu \mathrm{M}$ glipizide were prepared with each of these mobile phases and were applied using a $20 \mu \mathrm{L}$ injection volume, with the glipizide being monitored at $254 \mathrm{~nm}$. Other conditions and procedures, including the use of sodium nitrate as a nonretained solute, where the same as described for the other zonal elution competition studies.

\section{Theory}

\section{Frontal Analysis}

The method of frontal analysis was used to provide initial estimates for the binding constants and amount or types of binding sites for glipizide with normal HSA or glycated HSA [3942,53]. Fig. 2(a) shows the general format of this approach and some typical chromatograms that were produced by this method. In this type of experiment, a series of breakthrough curves were produced when known concentrations of glipizide were continuously applied to a column. The mean position of each breakthrough curve was then determined, which occurred around 4-8 min for the results that are given in Fig. 2(a).

In a system where relatively fast association and dissociation kinetics are present between the applied drug or analyte (A) and the immobilized protein or ligand (L), the mean position of the breakthrough curves can be related to the concentration of A, the association equilibrium constant(s) for this analyte with the ligand, and the moles of binding sites for the analyte in the column [39-42,53]. For example, if binding by A occurs at a single type of site on $\mathrm{L}$, this system can be described by the one-site binding model that is represented by Eqs. (1) and (2) [39-42,53].

$$
\begin{aligned}
& m_{\text {Lapp }}=\frac{m_{L} K_{a}[\mathrm{~A}]}{\left(1+K_{a}[\mathrm{~A}]\right)} \\
& \frac{1}{m_{\text {Lapp }}}=\frac{1}{K_{a} m_{L}[\mathrm{~A}]}+\frac{1}{m_{L}}
\end{aligned}
$$


In these equations, $m_{\text {Lapp }}$ refers to the moles of applied analyte that are required to reach the central point of the breakthrough curve at a given molar concentration of the analyte, [A]. The association equilibrium constant and total moles of active binding sites for this interaction are represented by $K_{a}$ and $m_{L}$, respectively. Eq. (2) is the double-reciprocal form of Eq. (1), which predicts that a linear relationship should be present in a plot of $1 / m_{L a p p}$ versus 1/[A] for this type of system. The value of $m_{L}$ in this plot is given by the inverse of the intercept, and $K_{a}$ can be obtained independently from $m_{L}$ by taking the ratio of the intercept over the slope.

Similar relationships can be used to describe more complex interactions, like the two-site system that is represented by Eqs. (3-4) [39-42,53].

$$
\begin{gathered}
m_{\text {Lapp }}=\frac{m_{L 1} K_{a 1}[\mathrm{~A}]}{\left(1+K_{a 1}[\mathrm{~A}]\right)}+\frac{m_{L 2} K_{a 2}[\mathrm{~A}]}{\left(1+K_{a 2}[\mathrm{~A}]\right)} \\
\frac{1}{m_{L a p p}}=\frac{1+K_{a 1}[\mathrm{~A}]+\beta_{2} K_{a 1}[\mathrm{~A}]+\beta_{2} K_{a 1}{ }^{2}[\mathrm{~A}]^{2}}{m_{L}\left\{\left(\alpha_{1}+\beta_{2}-\alpha_{1} \beta_{2}\right) K_{a 1}[\mathrm{~A}]+\beta_{2} K_{a 1}{ }^{2}[\mathrm{~A}]^{2}\right\}}
\end{gathered}
$$

In these equations, $K_{a 1}$ and $K_{a 2}$ are the association equilibrium constants for the two types of binding sites for A in the column [39-42,53]. The binding capacities of these sites are described by $m_{L 1}$ and $m_{L 2}$, respectively. The double-reciprocal form of Eq. (3) is shown in Eq. (4), where $a_{1}$ is the fraction of all binding sites for A that consist of the highest affinity site for $\mathrm{A}$, and $\beta_{2}$ is the ratio of the association equilibrium constants for the lower versus higher affinity sites, where $\beta_{2}=K_{a 2} / K_{a 1}$ and $K_{a 1}>K_{a 2}$ [39-42,53].

Results plotted according to Eq. (4) would be expected to give a non-linear response over a broad range of concentrations for A; this feature has been used in prior studies to determine whether multi-site interactions are taking place between an analyte and an immobilized protein [39-42,53]. These deviations are most apparent at high concentrations of [A], or low values of 1/[A]. However, a plot of $1 / m_{\text {Lapp }}$ versus $1 /[\mathrm{A}]$ in even a multi-site system can approach a linear response at low concentrations of $\mathrm{A}$, or high 1/[A] values, as is indicated by Eq. (5) [39-42,53].

$$
\lim _{[\mathrm{A}] \rightarrow 0} \frac{1}{m_{\text {Lapp }}}=\frac{1}{m_{L}\left(\alpha_{1}+\beta_{2}-\alpha_{1} \beta_{2}\right) K_{a 1}[\mathrm{~A}]}+\frac{\alpha_{1}+\beta_{2}^{2}-\alpha_{1} \beta_{2}^{2}}{m_{L}\left(\alpha_{1}+\beta_{2}-\alpha_{1} \beta_{2}\right)^{2}}
$$

If data from the linear range in a plot of $1 / m_{\text {Lapp }}$ versus $1 /[\mathrm{A}]$ are analyzed according to Eq. (5) for a multi-site system, the apparent association equilibrium constant that is obtained from the ratio of the intercept over the slope can provide an initial estimate for the association equilibrium constant for the highest affinity sites in this system [39-42,53]. 


\section{Zonal Elution Competition Studies}

Zonal elution can be used to determine the affinity of an analyte at a specific region on an immobilized binding agent [26,39-42,53]. This type of experiment involves the injection of a small plug of a site-specific probe onto the column, while a known concentration of the competing agent (e.g., the analyte) is applied in the mobile phase [39-42,53]. Examples of some typical chromatograms that are obtained in such an experiment are included in Fig. 2(b). In this current report, such a method generally provided results within 4 to 10 min of sample injection. In this type of experiment, the retention of the probe is examined as the concentration of the competing agent is varied [39-42,53]. This retention is usually expressed in terms of the retention factor $(k)$ for the probe, as is calculated from the measured mean retention time for the probe $\left(t_{R}\right)$ and the void time $\left(t_{M}\right)$ of the system, where $k=\left(t_{R}-t_{M}\right) / t_{M}[24]$.

The change in the value of $k$ as the concentration of the competing agent (I) is varied is next examined according to various binding models. For instance, these results can be analyzed by using Eq. (6) and looking at the fit of this equation to a plot of $1 / k$ versus the molar concentration of the competing agent, [I] [39-42,53].

$$
\frac{1}{k}=\frac{K_{a \mathrm{I}} V_{M}[\mathrm{I}]}{K_{a \mathrm{~A}} m_{L}}+\frac{V_{M}}{K_{a A} m_{L}}
$$

In this equation, the association equilibrium constants for the probe and competition agent at their site of competition are described by $K_{a \mathrm{~A}}$ and $K_{\mathrm{aI}}$. The term $V_{M}$ is the column void volume, while $m_{L}$ is the total active moles of the common binding site in the column. If a linear response with a positive slope is obtained for a plot that is made according to Eq. (6), the results can be said to follow a model in which the probe and competing agent are interacting directly at a single type of site on the immobilized binding agent. In addition, the ratio of the slope versus the intercept from this plot can be used, in a manner that is independent of $m_{L}$, to determine the association equilibrium constant for the competing agent at its site of direct competition with the probe [39-42,53].

Deviations from the linear response that is predicted by Eq. (6) can occur if the probe binds at multiple sites or if allosteric effects are present during the binding of the probe and competing agent $[26,39-42,56]$. Eq. (7) can be used to describe such data when the probe and competing agent are binding to two separate but allosterically-linked sites [24,26].

$$
\frac{k_{0}}{k-k_{0}}=\frac{1}{\beta_{\mathrm{I} \rightarrow \mathrm{A}^{-1}}} \bullet\left(\frac{1}{K_{a \mathrm{IL}}^{[\mathrm{I}]}}+1\right)
$$

In this equation, the association equilibrium constant for the competing agent is represented by $K_{a \mathrm{aL}}$. The term $k_{0}$ is the retention factor for the probe in the absence of the competing agent, and $k$ is the retention factor for the probe in the presence of a given concentration of the competing agent. The term $\beta_{\mathrm{I} \rightarrow \mathrm{A}}$ is the coupling constant for the allosteric effect of I on 
the interaction of A with the immobilized binding agent [24]. A plot of $k_{0} /\left(k-k_{0}\right)$ vs. $1 /[\mathrm{I}]$ according to Eq. (7) should result in a linear relationship for a simple allosteric interaction, where the slope and intercept can be used to determine the coupling constant $\left(\beta_{\mathrm{I} \rightarrow \mathrm{A}}\right)$ and the association equilibrium constant for the competing agent [24,26]. A value of $\beta_{\mathrm{I} \rightarrow \mathrm{A}}$ that is greater than 1 indicates a positive allosteric effect is occurring, while $0<\beta_{\mathrm{I} \rightarrow \mathrm{A}}<1$ indicates that a negative allosteric effect is present. A value of zero for $\beta_{\mathrm{I} \rightarrow \mathrm{A}}$ is obtained when direct competition is taking place between A and I, and no competition is represented by a value of $\beta_{\mathrm{I} \rightarrow \mathrm{A}}$ that is equal to 1 [24].

Competition studies can also be conducted by injecting a small plug of the analyte onto the column in the presence of known concentrations of a site-specific probe in the mobile phase [40]. The retention factor for an analyte that interacts with $n$ independent sites can be represented by the sum of the retention factors for these individual sites $\left(k_{1}\right.$ through $\left.k_{\mathrm{n}}\right)$, as is shown in Eq. (8). This equation can further be expanded into Eq. (9) if an agent is also present in the mobile phase that can compete at one or more of these sites [40].

$$
\begin{gathered}
k_{\mathrm{A}}=k_{1}+\ldots k_{n} \\
k_{\mathrm{A}}=\frac{K_{a \mathrm{~A} 1} m_{L 1}}{V_{M}\left(1+K_{a \mathrm{I} 1}[\mathrm{I}]\right)} \ldots+\frac{K_{a \mathrm{~A} n} m_{L 1}}{V_{M}\left(1+K_{a \mathrm{I} n}[\mathrm{I}]\right)}
\end{gathered}
$$

In Eq. (9), $K_{\mathrm{aA} 1}$ through $K_{a \mathrm{~A} n}$ are the association equilibrium constants for the analyte at site 1 through $n$, and $K_{\text {al1 }}$ through $K_{\text {aln }}$ are the association equilibrium constants for the competing agent at the same sites [40].

\section{Results and Discussion}

\section{Frontal Analysis using Normal HSA}

Frontal analysis was first used to examine the overall binding of glipizide, a sulfonylurea drug with limited solubility in aqueous solutions, to columns containing either normal HSA or in vitro glycated HSA. Examples of such experiments are shown in Fig. 2(a). Previous studies have found that other sulfonylurea drugs tend to interact with both normal HSA and glycated HSA at two general classes of sites: a set of moderate-to-high affinity sites and a larger group of weak affinity regions [26-28,30,31]. This result has also been obtained with glibenclamide, another sulfonylurea drug with limited solubility in water [31].

In this report, the results from frontal analysis experiments with glipizide were fit to various binding models. Fig. 3(a) shows the best-fit line that was obtained for glipizide and normal HSA when using a one-site model, as described by Eq. (1). This fit resulted in a correlation coefficient of $0.9979(n=12)$. The same data were also fit to a two-site model, as described by Eq. (3) and shown in Fig. 3(b). This model gave a slightly higher correlation coefficient of 0.9998 ( $n=12)$. Residual plots, as provided by the insets of Fig. 3, indicated that the data had a more random distribution about the best-fit line for the two-site model than for the 
one-site model. In addition, the sum of the squares of the residuals was lower for the twosite model vs. the one-site model (i.e., $3.6 \times 10^{-19}$ vs. $5.3 \times 10^{-18}$ ). The presence of multisite binding was confirmed by plotting the data according to Eq. (2) or Eq. (4), which resulted in deviations from a linear response at high glipizide concentrations (see Supplementary Material). All of these results indicated that the two-site model was a better description than the one-site model for the binding of glipizide with normal HSA. The use of higher order models (e.g., a three-site model) did not result in any improved fit to this data.

The two-site model was next used to estimate the average association equilibrium constants and moles of sites that were involved in the interactions of glipizide with normal HSA. The results of this fit gave a value for $K_{\mathrm{a} 1}$ of $2.4( \pm 0.8) \times 10^{5} \mathrm{M}^{-1}$ for the group of relatively high affinity sites and a value for $K_{\mathrm{a} 2}$ of $1.7( \pm 0.4) \times 10^{4} \mathrm{M}^{-1}$ for the lower affinity sites. A comparable estimate of $1.1( \pm 0.1) \times 10^{5} \mathrm{M}^{-1}$ was obtained for $K_{\mathrm{a} 1}$ from the linear region of a plot that was made according to Eq. (5) (see Supplementary Material). In addition, these $K_{\mathrm{a} 1}$ values were in the same general range as an overall association equilibrium constant of $3.7 \times 10^{5} \mathrm{M}^{-1}$ that has been reported for glipizide with normal HSA in solution, as determined by fluorescence spectroscopy at $37^{\circ} \mathrm{C}$ and in $\mathrm{pH} 7.4,0.10 \mathrm{M}$ phosphate buffer [34].

The $K_{a 1}$ values that were determined for the high affinity sites of glipizide were similar to or up to 3.4-fold larger than values that have been measured by frontal analysis for the high affinity sites of acetohexamide, tolbutamide, and gliclazide with normal HSA [26-28,30]. This strong binding by glipizide may be due to the large and relatively non-polar side chains that are present in glipizide [57], which have been proposed to be important in the interactions of this drug with HSA [34]. The $K_{a 1}$ values estimated for glipizide were up to an order of magnitude lower than the average $K_{a 1}$ that has been reported for the high affinity sites of normal HSA with glibenclamide, another second-generation drug [31]. However, glibenclamide is known to bind to an additional high affinity site (i.e., the digitoxin site) that significantly increases its overall affinity for normal HSA when compared with many other sulfonylurea drugs [31].

The normal HSA column was estimated through the frontal analysis data to have a binding capacity of $8.9( \pm 3.0) \times 10^{-9} \mathrm{~mol}$, or $2.9( \pm 0.1) \times 10^{-7} \mathrm{~mol} / \mathrm{g}$ silica, for glipizide at its highest affinity sites and $6.0( \pm 0.3) \times 10^{-8} \mathrm{~mol}$, or $1.9( \pm 0.1) \times 10^{-6} \mathrm{~mol} / \mathrm{g}$ silica, at its lower affinity sites. Based on the known protein content of the column, the specific activities for these sites were $0.24( \pm 0.08)$ and $1.6( \pm 0.1) \mathrm{mol} / \mathrm{mol} \mathrm{HSA}$, respectively. These values indicated that at least one major binding site was involved in the higher affinity interactions, with a larger group of sites taking part in the weaker affinity interactions. These results and overall trends are comparable to those that have been obtained in previous frontal analysis studies involving normal HSA and other sulfonylurea drugs [26-28,30,31].

\section{Frontal Analysis using Glycated HSA}

When examining the interactions of glipizide with two samples of in vitro glycated HSA (i.e., gHSA1 and gHSA2), the two-site model again gave a better fit for the frontal analysis data than a one-site model. The correlation coefficients obtained for the frontal analysis data with glipizide and gHSA1 were $0.9964(n=12)$ for the one-site model and $0.9997(n=12)$ 
for the two-site model. Similar results were obtained for gHSA2, with correlation coefficients of $0.9978(n=12)$ for the one-site model and $0.9999(n=12)$ for the two-site model. The residual plots for both types of glycated HSA gave a more random distribution of the data about the best-fit line for the two-site model than for the one-site model. The data for gHSA1 and gHSA2 also had a lower sum of the squares of the residuals for the two-site model than the one-site model (i.e., $5.6 \times 10^{-19}$ vs. $6.9 \times 10^{-18}$ for gHSA1 and $4.1 \times 10^{-19}$ vs. $1.9 \times 10^{-18}$ for gHSA2. These results confirmed glipizide also interacted with the glycated HSA samples through a two-site model.

Table 1 includes the binding parameters that were obtained for glipizide with the glycated HSA samples when using the two-site model. The average association equilibrium constants that were measured for glipizide at its higher and lower affinity sites on gHSA1 were 2.8 $( \pm 1.1) \times 10^{5} \mathrm{M}^{-1}$ and $2.5( \pm 0.5) \times 10^{4} \mathrm{M}^{-1}$, respectively. The corresponding values for gHSA2 were $6.0( \pm 2.2) \times 10^{5} \mathrm{M}^{-1}$ and $3.7( \pm 0.2) \times 10^{4} \mathrm{M}^{-1}$. The average association equilibrium constants that are shown in Table 1 for the highest affinity sites were 1.2-fold higher for gHSA1 and 2.5-fold higher for gHSA2 than the value that was measured for normal HSA. The apparent increase in affinity for gHSA1 was not statistically significant at the $95 \%$ confidence level; however, the increase seen for gHSA2 was significant. Previous studies involving other sulfonylurea drugs have noted an increase in the average $K_{a}$ at the highest affinity sites for these drugs on glycated HSA versus normal HSA [27,28,30,31].

It has been suggested that such changes in affinity, if present, may be a result of alterations in the amount or types of glycation products that are present at or near drug binding regions as the level of HSA glycation is varied [58,59]. For instance, studies using mass spectrometry and digests of HSA have confirmed that significant levels of glycation-related modifications can occur at or near the major drug binding sites of moderately and highly glycated HSA [59]. Examples of modification sites that are present at or near Sudlow site I include K199, K150, K160, K275, K281, R209, and R257, while possible modification sites that are located at or near Sudlow site II include K378, K439, and K428 [59]. It has also been shown that the modification pattern can change with the extent of glycation [58,59]. For instance, differences have been seen in the types of modifications that are present at residues K199, R197, and R428 for a moderately glycated sample of HSA versus highly glycated samples of HSA [58].

The specific activities for the columns containing glycated HSA were determined from the measured binding capacities and protein contents for these columns. The higher and lower affinity sites in the gHSA1 column had binding capacities of $9.7( \pm 3.8) \times 10^{-9}$ and 6.3 $( \pm 0.2) \times 10^{-8} \mathrm{~mol}$, or $3.1( \pm 1.2) \times 10^{-7} \mathrm{~mol} / \mathrm{g}$ silica and $2.0( \pm 0.1) \times 10-6 \mathrm{~mol} / \mathrm{g}$ silica, respectively, with specific activities of $0.30( \pm 0.11)$ and $2.0( \pm 0.1) \mathrm{mol} / \mathrm{mol}$ gHSA1. The gHSA2 column had binding capacities for the same sites of $4.2( \pm 1.2) \times 10^{-9}$ and 5.9 $( \pm 0.1) \times 10^{-8} \mathrm{~mol}$, or $1.4( \pm 0.4) \times 10^{-7} \mathrm{~mol} / \mathrm{g}$ silica and $1.9( \pm 0.1) \times 10^{-6} \mathrm{~mol} / \mathrm{g}$ silica, with specific activities of $0.12( \pm 0.03)$ and $1.7( \pm 0.1) \mathrm{mol} / \mathrm{mol}$ gHSA2. These results were similar to those obtained for the normal HSA column and in prior work with other sulfonylurea drugs [26-28,30,31]. These results again indicated that at least one type of site was involved in the higher affinity interactions and that more sites were involved in the weaker affinity interactions. 


\section{Competition Studies at Sudlow Site II}

Zonal elution competition studies were used to help identify the binding sites for glipizide on HSA and to measure the affinity of glipizide at these sites. Sudlow site II has been found in previous work to be one of the regions that often takes part in the moderate-to-high affinity interactions of other sulfonylurea drugs with normal HSA and glycated HSA [26$28,30,31]$. It has recently been determined in solution-phase displacement experiments that glipizide also binds to Sudlow site II on normal HSA [34]. In this current report, interactions at Sudlow site II were examined by using L-tryptophan as a probe for this site [26-31].

Competition studies between L-tryptophan with glipizide were carried out on columns that contained normal HSA or glycated HSA. In these studies, L-tryptophan was injected as the probe and various concentrations of glipizide were placed into the mobile phase. The data were then analyzed according to Eq. (6), as illustrated in Fig. 4(a). A linear response was observed for glipizide on all of the normal HSA or glycated HSA columns, with correlation coefficients that ranged from 0.9137 to $0.9643(n=8)$. It was concluded from these results that L-tryptophan and glipizide had direct competition at Sudlow site II on both normal HSA and glycated HSA.

Table 2 shows the association equilibrium constants for glipizide that were measured at Sudlow site II on these various columns. These values that were obtained were $1.1( \pm 0.1) \times$ $10^{4} \mathrm{M}^{-1}$ for normal HSA, $1.2( \pm 0.1) \times 10^{4} \mathrm{M}^{-1}$ for gHSA1, and $1.4( \pm 0.2) \times 10^{4} \mathrm{M}^{-1}$ gHSA2. The change in affinity between normal HSA and gHSA1 (9\%) was not statistically different at either the $95 \%$ or $90 \%$ confidence level. However, a significant increase (at both confidence levels) of $27 \%$ or $17 \%$ was observed in this affinity at Sudlow site II when going from normal HSA or gHSA1 to gHSA2. In addition, these affinity values were approximately 3.5 - to 12 -fold lower than association equilibrium constants that have been measured for other first- or second-generation sulfonylurea drugs at Sudlow site II of normal HSA $[27,28,30,31]$.

It has been suggested based on solution-phase displacement studies that Sudlow site II is the major binding site for glipizide on normal HSA [34]. However, the association equilibrium constants measured here for glipizide at Sudlow site II of normal HSA and glycated HSA were an order of magnitude lower than the binding constants that were estimated for the highest affinity sites, as determined by frontal analysis (see Table 1 and Supplementary Material). This comparison indicated that although glipizide was binding to Sudlow site II, some other region was also present that had an even higher affinity for this drug. The location of this additional site was explored in the following sections by using probes for other regions of HSA.

\section{Competition Studies at the Digitoxin Site}

The digitoxin site of HSA has been observed in prior work to have strong binding to the sulfonylurea drug glibenclamide [31]. As a result, competition studies were also conducted to examine the binding of glipizide at this site and by using digitoxin as a site-specific probe [22]. Injections of digitoxin onto the normal HSA column and in the presence of various concentrations of glipizide gave only a random variation in retention of $\pm 6.3 \%$, as 
demonstrated by a plot that was made according to Eq. (6) in Fig. 4(b). Similar results were obtained for the columns containing gHSA1 or gHSA2, which gave random variations in the retention for digitoxin of $\pm 4.6 \%$ and $\pm 9.5 \%$, respectively. These results indicated that glipizide was not interacting with the digitoxin site of either normal HSA or glycated HSA.

\section{Competition Studies at Sudlow Site I}

Competition studies were next carried out at Sudlow site I by using $R$-warfarin as a sitespecific probe for this binding region [20,24,29]. Previous work involving other sulfonylurea drugs have consistently noted that these drugs have direct competition for warfarin at this site and that this region has moderate-to-high affinity interactions for this class of drugs [26$28,30,31]$. This site has further been identified, through solution-phase displacement studies, as a binding region for glipizide on normal HSA [34].

Data from competition studies using glipizide as a mobile phase additive and $R$-warfarin as an injected probe compound were plotted according to Eq. (6). An example of such a plot is shown in Fig. 5(a), as generated using the normal HSA column. In previous studies with other sulfonylurea drugs, linear behavior and a positive slope has been seen for such plots, indicating that $R$-warfarin was competing directly with these drugs [26-28,30,31]. The corresponding plot for glipizide was also linear with a positive slope at gliclazide concentrations up to about $5 \mu \mathrm{M}$, with deviations from linearity occurring at higher glipizide concentrations. Similar results were obtained for the glycated HSA columns. These results suggested that glipizide was in direct competition with $R$-warfarin at Sudlow site I at low glipizide concentrations. Such behavior agreed with the results from prior solution-phase displacement studies [34].

The deviations from linearity that were seen in plots like Fig. 5(a) could have been produced by an allosteric interaction between the injected probe and glipizide as the concentration of this drug was increased $[26,56]$. This effect may be related to the ability of glipizide to induce a conformational change as a result of binding by this drug to HSA, as has been proposed from solution-phase studies [34]. The presence of an allosteric interaction was tested by plotting the zonal elution data according to Eq. (7). As is shown in Fig. 5(b), the resulting graph of $k_{0} /\left(k-k_{0}\right)$ vs. 1/[Glipizide] for the normal HSA column gave a linear relationship with a negative slope at low values of $1 /[$ Glipizide]. The glycated HSA columns gave similar linear relationships. Deviations from linearity were observed at high values of 1/[Glipizide], as demonstrated in Fig. 5(b). These deviations occurred at the same glipizide concentrations that were in the linear range of plots made according to Eq. (6), as based on a direct competition model. The linear region in plots made according to Eq. (7) gave correlation coefficients of -0.7452 to $-0.9975(n=4)$ for the normal HSA and glycated HSA columns.

The linear fits obtained with Eq. (7) were used to estimate the association equilibrium constants for glipizide at the site that was taking part in this allosteric interaction (see Table 2). The same fits were used to find the coupling constants for the allosteric effect of glipizide on the binding of $R$-warfarin with normal HSA or glycated HSA. The association equilibrium constant that was measured for glipizide at its interaction region on normal HSA was $3.9( \pm 0.2) \times 10^{5} \mathrm{M}^{-1}$. This value was similar to the overall association equilibrium 
constant that has been reported for the binding of glipizide with normal HSA in solution [34] and the value obtained in this current report for the high affinity regions of glipizide with normal HSA. The coupling constant that was measured for this site with $R$-warfarin was $0.60( \pm 0.03)$, with the latter value representing a negative allosteric effect. The association equilibrium constants for glipizide at the corresponding interaction sites on gHSA1 and gHSA2 were $3.2( \pm 0.2) \times 10^{5} \mathrm{M}^{-1}$ and $3.9( \pm 0.3) \times 10^{5} \mathrm{M}^{-1}$, with coupling constants of $0.66( \pm 0.05)$ and $0.62( \pm 0.05)$, respectively. There was an $18 \%$ decrease in affinity for glipizide and this interaction in going from normal HSA to gHSA1, and a similar increase between gHSA 1 and gHSA2. Both of these changes were significant at the $95 \%$ confidence interval. No changes in affinity were observed between normal HSA and gHSA2. In addition, all of the coupling constants measured between glipizide and warfarin were statistically equivalent at the $95 \%$ confidence interval.

Binding by glipizide at Sudlow site I was confirmed by carrying out a reverse competition study. This was done by injecting a small amount of glipizide as the probe and by using racemic warfarin as the competing agent in the mobile phase. The measured retention factor for glipizide was also corrected for the binding of this drug to Sudlow site II and to its weak affinity regions; this was accomplished by using Eq. (9) with the results of the competition studies at Sudlow site II and the binding constants in Table 1 for the weak affinity sites that were measured by frontal analysis. The resulting corrected retention factors gave linear plots when analyzed according to Eq. (6) (see Supplementary Material), as would be expected if direct competition were occurring between glipizide and warfarin in the region of Sudlow site I. Furthermore, the association equilibrium constants that were estimated for glipizide at Sudlow site I from these results were consistent with the range of values that were obtained when using $R$-warfarin as the injected probe and glipizide as the competing agent.

\section{Competition Studies at the Tamoxifen Site}

Because some allosteric effects were observed for glipizide at Sudlow site I, additional experiments were used to explore whether glipizide had similar effects at other sites on HSA. The tamoxifen site of HSA was of particular interest since previous studies have shown that Sudlow site I and this site are allosterically-linked [24,60,61]. These studies were originally carried out by injecting tamoxifen as a site-specific probe [24] and placing glipizide as a competing agent in the mobile phase. When the results were analyzed according to Eq. (6) and a direct-competition model, the plot obtained for normal HSA gave a non-linear response with a negative slope, as shown in Fig. 6(a). This indicated that a positive allosteric interaction was present between glipizide and tamoxifen [24], with similar trends being seen for glycated HSA.

The same competition data were analyzed by using Eq. (7). As shown in Fig. 6(b), a linear fit was noted for a plot of $k_{0} /\left(k-k_{0}\right)$ vs. 1/[Glipizide] for the normal HSA column. The same behavior was seen on the glycated HSA columns. These plots had correlation coefficients of 0.9976 to $0.9988(n=7)$. These fits were used to find the association equilibrium constant for glipizide at the site that had an allosteric effect on the tamoxifen site. The results are included in Table 2. The association equilibrium constant measured for glipizide when using normal HSA was $4.1( \pm 0.9) \times 104 \mathrm{M}^{-1}$, and the coupling constant of this region with the 
tamoxifen site was $8.0( \pm 1.7)$. gHSA1 and gHSA2 had association equilibrium constants of $7.8( \pm 1.1) \times 10^{4} \mathrm{M}^{-1}$ and $5.2( \pm 1.3) \times 10^{4} \mathrm{M}^{-1}$, respectively, for glipizide during this allosteric interaction, as well as coupling constants of $5.1( \pm 0.7)$ and $6.1( \pm 1.5)$. The 1.9fold increase in the affinity between normal HSA and gHSA1 was significant at the $95 \%$ confidence interval. The 1.3-fold increase in affinity between normal HSA and gHSA2 was not significant at the 95\% confidence level but was significant at the $90 \%$ confidence level. Significant differences at the $95 \%$ confidence level were also present between the coupling constants for normal HSA and gHSA1or gHSA2.

Reverse competition studies were conducted by using glipizide as the injected probe and tamoxifen as the competing agent. The retention factors for glipizide were again corrected for the binding of this drug at Sudlow site II and at its weak affinity regions, as described in the previous section. A plot using these corrected values was made according to Eq. (6). This plot resulted in a non-linear relationship (see Supplementary Material) which further indicated that positive allosteric interactions where occurring between the binding region for glipizide on HSA and the tamoxifen site. Similar results were obtained for glycated HSA. The same type of positive allosteric effect on tamoxifen has been noted to occur during the binding of warfarin at Sudlow site I of HSA [24].

\section{Conclusion}

This report utilized HPAC to examine the binding of glipizide to normal HSA and HSA with various levels of in vitro glycation. The results of frontal analysis experiments indicated that glipizide was interacting with both normal HSA and glycated HSA at a series of relatively high affinity regions that had average association equilibrium constants in the range of 2.4$6.0 \times 10^{5} \mathrm{M}^{-1}$ at $\mathrm{pH} 7.4$ and $37^{\circ} \mathrm{C}$. These values were in good agreement with a previous estimate that has been made for the overall affinity of glipizide with normal HSA in solution [34]. This drug was also found to have a large set of weak affinity regions that had association equilibrium constants in the range of $1.7-3.7 \times 10^{4} \mathrm{M}^{-1}$.

Zonal elution competition studies were used to provide more detailed information on the interactions of glipizide with specific sites of normal HSA or glycated HSA. Fig. 7 summarizes the results of these experiments. This drug was found to bind to Sudlow site II with an affinity of 1.1-1.2 $\times 10^{4} \mathrm{M}^{-1}$ for both normal HSA and glycated HSA. The same proteins had estimated affinities of 3.2-3.9 $\times 10^{5} \mathrm{M}^{-1}$ for normal HSA and glycated HSA, which agreed with the values for the high affinity regions that were obtained by frontal analysis. Up to an $18 \%$ decrease in the affinity for glipizide was observed at Sudlow site I in going from normal HSA to glycated HSA, while up to a $27 \%$ increase was noted at Sudlow site II. Glipizide had no appreciable interactions at the digitoxin site and was found to have positive allosteric effect for the binding of tamoxifen at its site on HSA. A negative allosteric effect was also observed between glipizide and the binding of $R$-warfarin at Sudlow site I.

The results from this study demonstrate how HPAC can be used to examine the overall binding affinity and site-specific binding for drugs with relatively complex interactions with proteins, including modified proteins such as glycated HSA. This included the use of this method to detect direct competition, no competition and either positive or negative allosteric 
interactions between a drug such as glipizide and probes for various binding regions on these proteins. It was also shown how a combination of traditional zonal elution competition studies and reverse competition studies could be used together to help examine mixed-mode interactions, such as occurred between warfarin and glipizide at Sudlow site I.

In addition, the results from this study show how glycation, at levels comparable to those present in diabetes, can alter the binding of drugs with HSA. The type of binding data that was obtained in this report should be useful in future work in determining how the effective dosage of a drug like glipizide may be affected as the concentration of blood glucose and levels of protein glycation change in diabetic patients [9,25-31]. In addition, the methods that were used in this study are not limited to glipizide and normal HSA or glycated HSA, but could also be applied to other drugs or modified proteins. These features should make this approach, and the data it can provide, of interest in areas such as biointeraction studies, the screening of drug candidates, and personalized medicine [10,32].

\section{Supplementary Material}

Refer to Web version on PubMed Central for supplementary material.

\section{ACKNOWLEDGEMENTS}

This work was funded by the NIH under grant R01 DK069629. R. Matsuda was supported under a fellowship through the Molecular Mechanisms of Disease program at the University of Nebraska.

\section{References}

1. Centers for Disease Control and Prevention (2011) National diabetes fact sheet, 2011, Centers for Disease Control and Prevention, Atlanta

2. International Diabetes Federation (2011) IDF Diabetes Atlas, 5th edn., International Diabetes Federation, Brussels, chap 2.

3. Mendez DL, Jensen RA, McElroy LA, Pena JM, Esquerra RM (2005) Arch Biochem Biophys 444:92-99 [PubMed: 16309624]

4. Colmenarejo G (2003) Med Res Rev 23:275-301 [PubMed: 12647311]

5. Koyama H, Sugioka N, Uno A, Mori S, Nakajima K (1997) Biopharm Drug Dispos 18:791-801 [PubMed: 9429743]

6. Garlick RL, Mazer JS (1983) J Biol Chem 258:6142-6146 [PubMed: 6853480]

7. Iberg N, Fluckiger R (1986) J Biol Chem 261:13542-13545 [PubMed: 3759977]

8. Nakajou K, Watanabe H, Kragh-Hansen U, Maruyama T, Otagiri M (2003) Biochim Biophys Acta 1623:88-97 [PubMed: 14572906]

9. Furusyo N, Hayashi J (2013) Biochim Biophys Acta 1830:5509-5514 [PubMed: 23673238]

10. Anguizola J, Matsuda R, Barnaby OS, Joseph KS, Wa C, Debolt E, Koke M, Hage DS (2013) Clin Chim Acta 425:64-76 [PubMed: 23891854]

11. Roohk HV, Zaidi AR (2008) J Diabetes Sci Technol 2:1114-1121 [PubMed: 19885300]

12. Peters T, Jr (1996) All about albumin: biochemistry, genetics and medical applications Academic, San Diego

13. Clinical Guide to Laboratory Tests (1990) Tietz NW (ed), 2nd edn., Saunders, Philadelphia

14. Otagiri M (2005) Drug Metab Pharmacokinet 20:309-323 [PubMed: 16272748]

15. Curry S, Mandelkow H, Brick P, Franks N (1998) Nature Struct. Biol 5:827-535 [PubMed: 9731778]

16. Ascoli GA, Domenici E, Bertucci C (2006) Chirality 18:667-679 [PubMed: 16823814] 
17. Simard JR, Zunszain PA, Ha CE, Yang JS, Bhagavan NV, Petitpas I, Curry S, Hamilton JA (2005) Proc Natl Acad Sci USA 102:17958-17963 [PubMed: 16330771]

18. Sudlow G, Birkett J, Wade DN (1975) Mol Pharmacol 11:824-832 [PubMed: 1207674]

19. Sudlow G, Birkett J, Wade DN (1976) Mol Pharmacol 12:1052-1061 [PubMed: 1004490]

20. Loun B, Hage DS (1994) Anal Chem 66:3814-3822 [PubMed: 7802261]

21. Yang J, Hage DS (1993) J Chromatogr 645:241-250 [PubMed: 8408417]

22. Sengupta A, Hage DS (1999) J Chromatogr B 725:91-100

23. Hage DS, Sengupta A (1998) Anal Chem 70:4602-4609 [PubMed: 9823719]

24. Chen J, Hage DS (2006) Anal Chem 78:2672-2683 [PubMed: 16615779]

25. Jakoby MG, Covey DF, Cistola DP (1995) Biochemistry 34:8780-8787 [PubMed: 7612618]

26. Joseph KS, Hage DS (2010) J Chromatogr B 878:1590-1598

27. Joseph KS, Anguizola J, Jackson AJ, Hage DS (2010) J Chromatogr B 878:2775-2781

28. Joseph KS, Anguizola J, Hage DS (2011) J Pharm Biomed Anal 54:426-432 [PubMed: 20880646]

29. Joseph KS, Hage DS (2010) J Pharm Biomed Anal 53:811-818 [PubMed: 20537832]

30. Matsuda R, Anguizola J, Joseph KS, Hage DS (2011) Anal Bioanal Chem 401:2811-2819 [PubMed: 21922305]

31. Matsuda R, Anguizola J, Joseph KS, Hage DS (2012) J Chromatogr A 1265:114-122 [PubMed: 23092871]

32. Anguizola J, Joseph KS, Barnaby OS, Matsuda R, Alvarado G, Clarke W, Cerny RL, Hage DS (2013) Anal Chem 85:4453-4460 [PubMed: 23544441]

33. Jackson AJ, Anguizola J, Pfaunmiller EL, Hage DS (2013) Anal Bioanal Chem 405:5833-5841 [PubMed: 23657448]

34. Seeder N, Kanojia M (2009) Cent Eur J Chem 7:96-104

35. Melander A (2004) Diabetes 53:S151-S155 [PubMed: 15561903]

36. Tan Z, Zhang J, Wu J, Fang L, He Z (2009) AAPS PharmSciTech 10:967-976 [PubMed: 19636711]

37. Hansch C, Leo A, Hoekman (1995) Exploring QSAR - hydrophobic, electronic, and steric constants, American Chemical Society, Washington DC

38. Wishart DS, Knox C,Guo A, Cheng D, Shrivastava S, Tzur D, Gautum B, Hassanali M (2008) Nucleic Acid Res 36:D901-D906 [PubMed: 18048412]

39. Schiel JE, Joseph KS, Hage DS (2010) Biointeraction affinity chromatography. In: Grinsberg N, Grushka E (eds) Advances in Chromatography, Taylor \& Francis, New York, chap 4

40. Hage DS (2002) J Chromatogr B 768:3-30

41. Patel S, Wainer IW, Lough WJ (2006) In: Hage DS (ed) Handbook of affinity chromatography, 2nd edn. CRC, Boca Raton, chap 24

42. Hage DS, Anguizola J, Barnaby O, Jackson A, Yoo MJ, Papastavros E, Pfaunmiller E, Sobansky M, Tong Z (2011) Curr Drug Metabolism 12:313-328

43. Lapolla A, Fedele D, Reitano R, Arico NC, Seraglia R, Traldi P, Marotta E, Tonani R (2004) J Am Soc Mass Spectrom 15:496-509 [PubMed: 15047055]

44. Ney KA, Colley KJ, Pizzo SV (1981) Anal Biochem 118:294-300 [PubMed: 7337226]

45. Pfaunmiller E, Moser AC, Hage DS (2012) Methods 56:130-135 [PubMed: 21907805]

46. Walters RR (1982) J Chromatogr A 249:19-28

47. Larson PO (1984) Methods Enzymol 104:212-223 [PubMed: 6371445]

48. Kim HS, Hage DS (2006) In: Hage DS (ed) Handbook of affinity chromatography, 2nd edn. CRC, Boca Raton, chap 3

49. Wa C, Cerny RL, Hage DS (2006) Anal Chem 78:7967-7977 [PubMed: 17134129]

50. Joseph KS, Moser AC, Basiaga S, Schiel JE, Hage DS (2009) 1216:3492-3500

51. Yalkowsky SH, Dannenfelser RM (1992) Aquasol database of aqueous solubility, ver 5, University of Arizona, Tuscon

52. Teko IV, Thanchuk VY, Kasheva TN, Villa AE (2001) J Chem Inf Comput Sci 41:1488-1493

[PubMed: 11749573] 
53. Matsuda R, Anguizola J, Hoy KS, Hage DS (2014) Methods, in press

54. Conrad ML, Moser AC, Hage DS (2009) J Sep Sci 32:1145-1155 [PubMed: 19296478]

55. Jamzad S, Rassihi R (2006) AAPS PharmSciTech 7:E17-E22 [PubMed: 16584147]

56. Tweed SA (1997) Effects of heterogeneity on the characterization of chromatographic stationary phases Ph.D. dissertation, University of Nebraska, Lincoln

57. Crooks MJ, Brown KF (1974) J Pharm Pharmacol 26:304-311 [PubMed: 4153105]

58. Barnaby OS, Cerny RL, Clarke W, Hage DS (2011) Clin Chim Acta 412:277-285 [PubMed: 21034726]

59. Barnaby OS, Cerny RL, Clarke W, Hage DS (2011) Clin Chim Acta 412:1606-1615 [PubMed: 21601565]

60. Sjoholm I (1986) In Reindenberg MM, Erill S (eds) Drug-protein binding, New York, chap 4

61. Sengupta A, Hage DS (1999) Anal Chem 17:3821-3827 


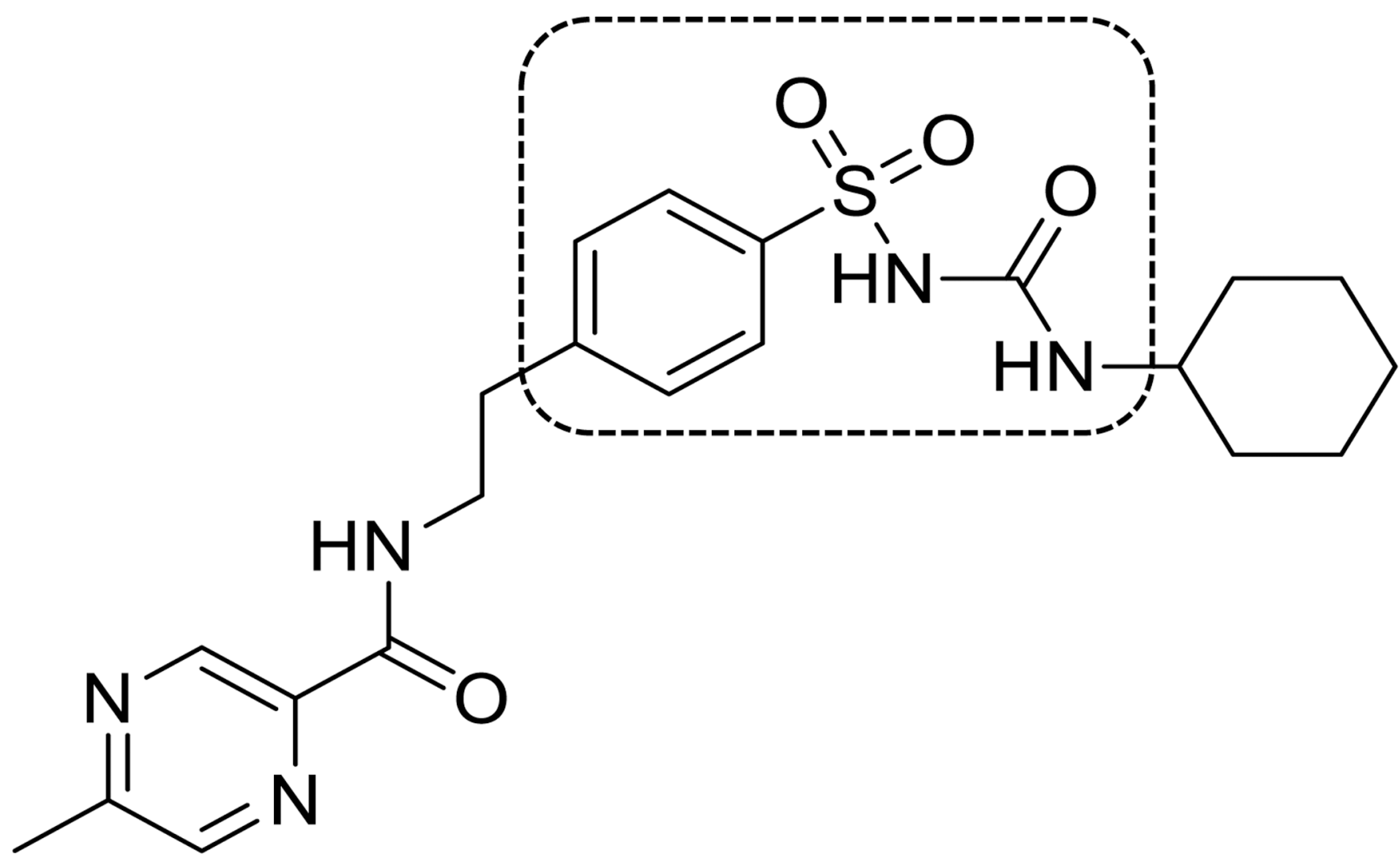

Figure 1.

Structure of glipizide. The section in the dashed box shows the common core structure of all sulfonylurea drugs. 


\section{(a) Frontal Analysis}

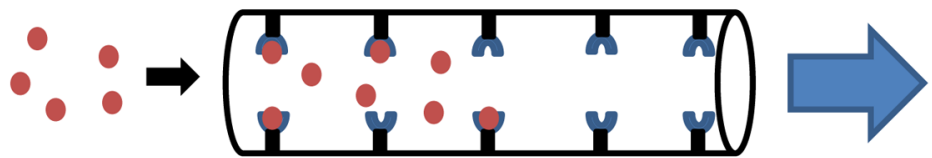

Continuous application

of target solute

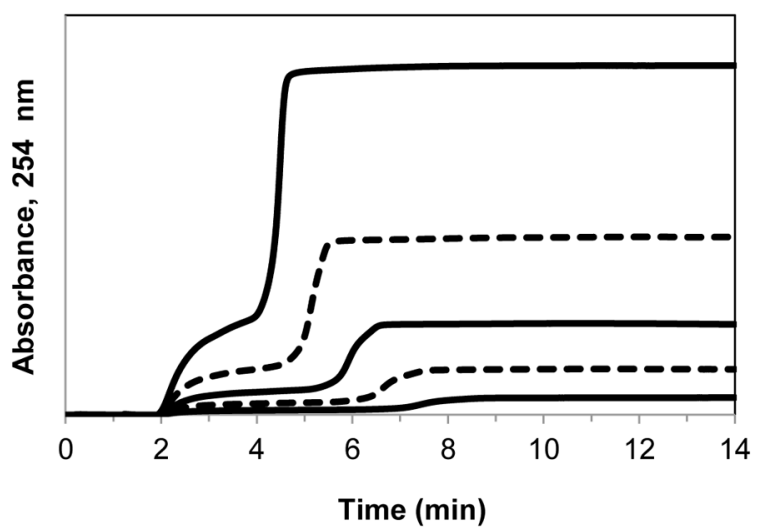

(b) Zonal Elution Competition Study
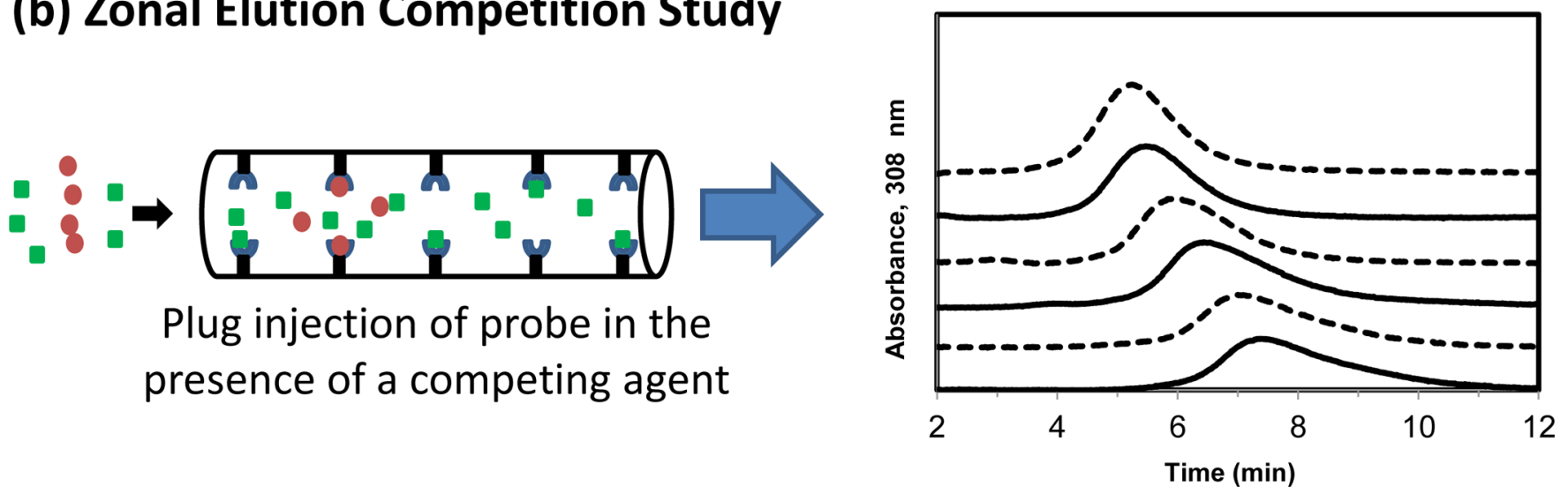

Figure 2.

Typical experimental formats and results for (a) frontal analysis and (b) zonal elution competition studies. These results were obtained for glipizide on $2.0 \mathrm{~cm} \times 2.1 \mathrm{~mm}$ i.d. columns containing normal HSA. The results in (a) are for glipizide concentrations of 20, $10,5,2.5$, or $1 \mu \mathrm{M}$ (top-to-bottom). The results in (b) were obtained using $R$-warfarin as an injected site-specific probe for Sudlow site I and in the presence of glipizide concentrations in the mobile phase of $20,10,5,2.5$, or $1 \mu \mathrm{M}$ (left-to-right). Other experimental conditions are given in the text. 

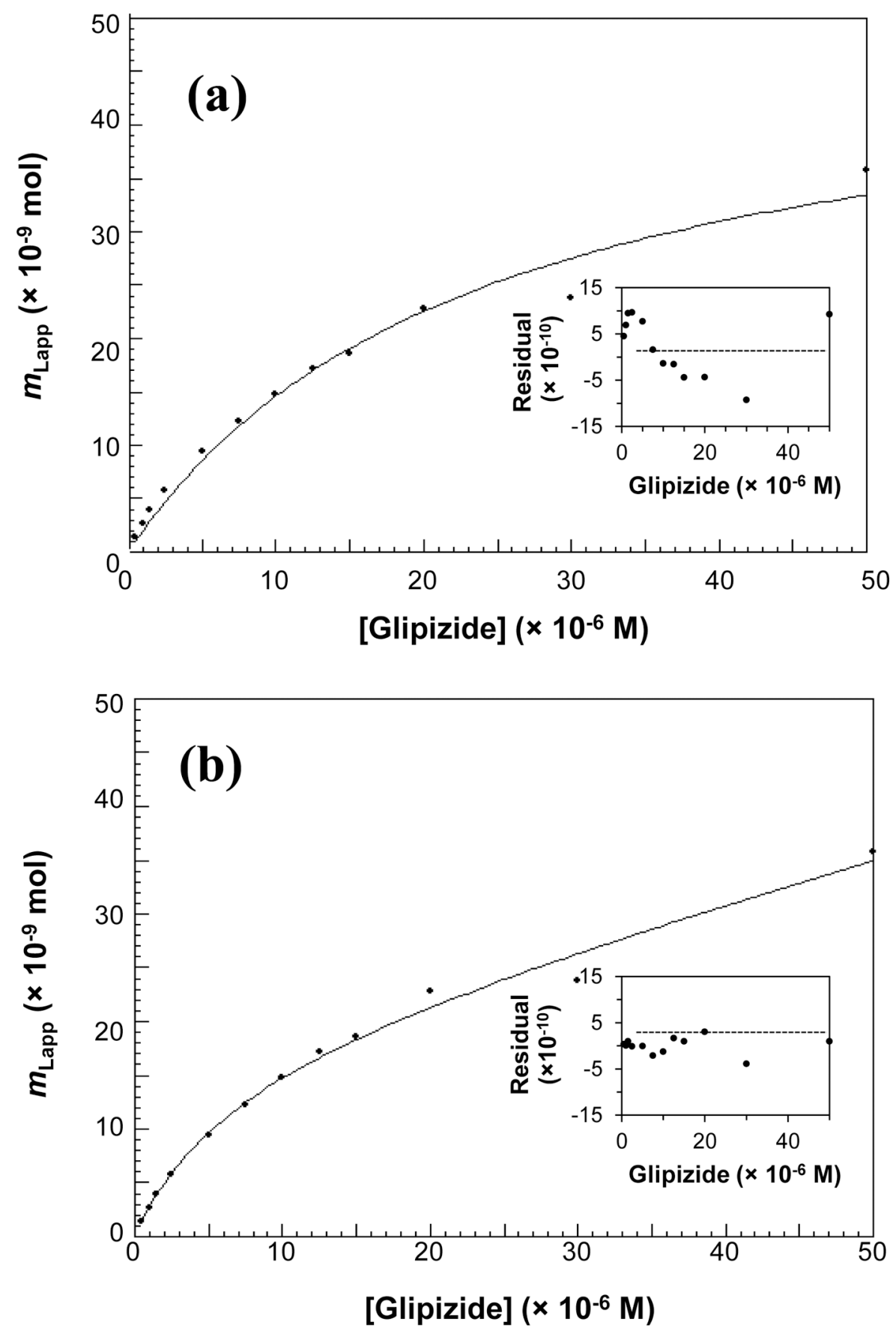

Figure 3.

Analysis of frontal analysis data for the binding of glipizide with normal HSA by using (a) a one-site model or (b) a two-site model. These results are for twelve solutions of glipizide with concentrations ranging from 0.5 to $50 \mu \mathrm{M}$ that were applied to a $2.0 \mathrm{~cm} \times 2.1 \mathrm{~mm}$ i.d. normal HSA column. Other experimental conditions are given in the text. The insets in (a) and (b) show the residual plots for the fits of the data to the given binding models. Each data point is the average of four values, with relative standard deviations ranging from $\pm 0.3-$ $4.6 \%$. 

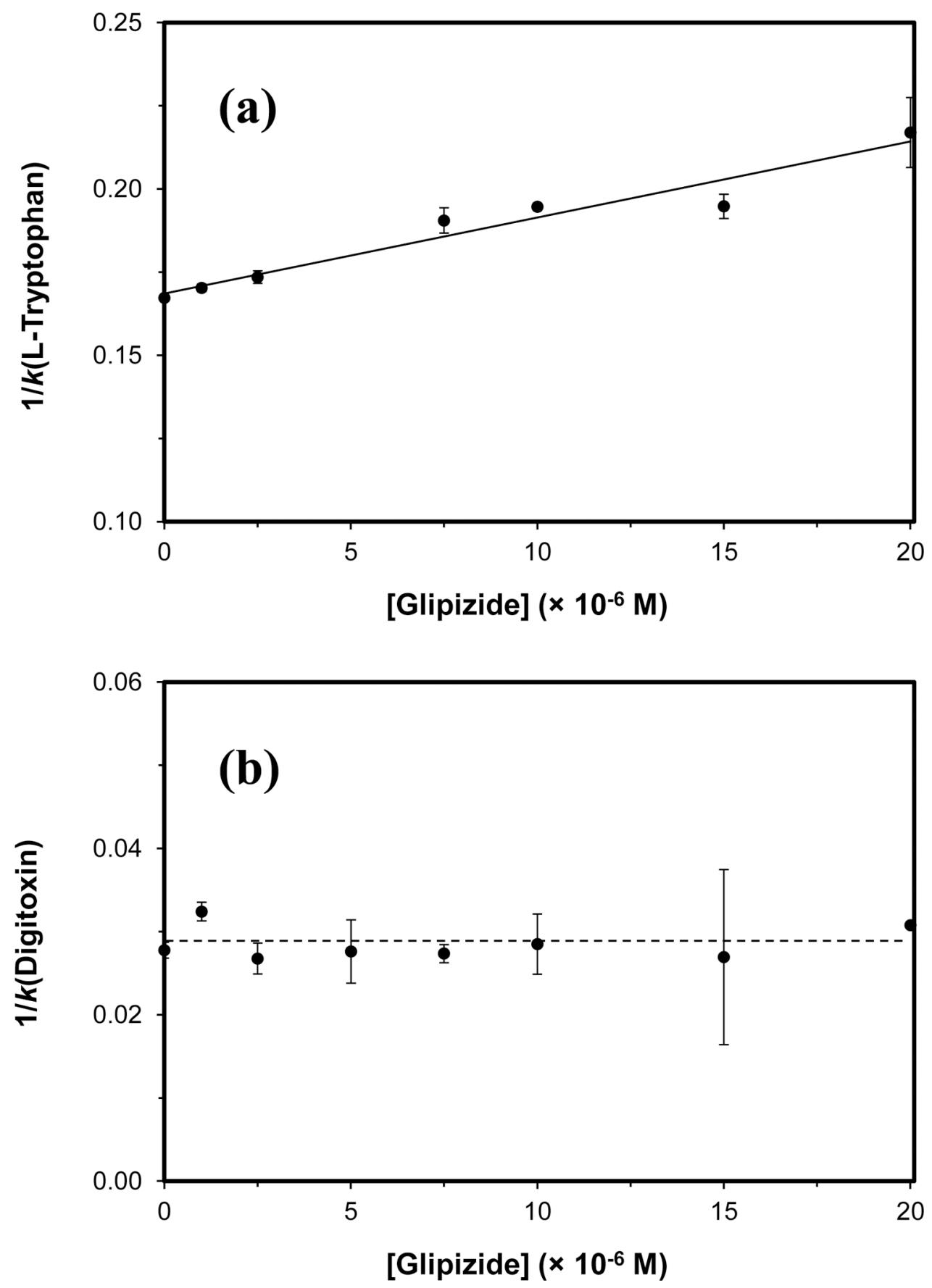

Figure 4.

Plots prepared according to Eq. (6) for zonal elution competition data obtained for glipizide and using (a) L-tryptophan as an injected probe for Sudlow site II on a $2.0 \mathrm{~cm} \times 2.1 \mathrm{~mm}$ i.d. gHSA2 column or (b) digitoxin as an injected probe for the digitoxin site on a $2.0 \mathrm{~cm} \times 2.1$ $\mathrm{mm}$ i.d. normal HSA column. Other experimental conditions are given in the text. The equation for the best-fit line in (a) is $y=\left[2.2( \pm 0.3) \times 10^{3}\right] x+[0.2( \pm 0.1)]$, with a correlation coefficient of $0.9707(n=7)$. The dashed reference line in (b) is the result expected for a system with no competition between the injected probe and competing agent. 
The error bars represent a range of \pm 1 S.D. Each point is the average of four values with relative standard deviations that ranged from (a) $\pm 0.5-4.8 \%$ or (b) $\pm 0.5-5.0 \%$. 

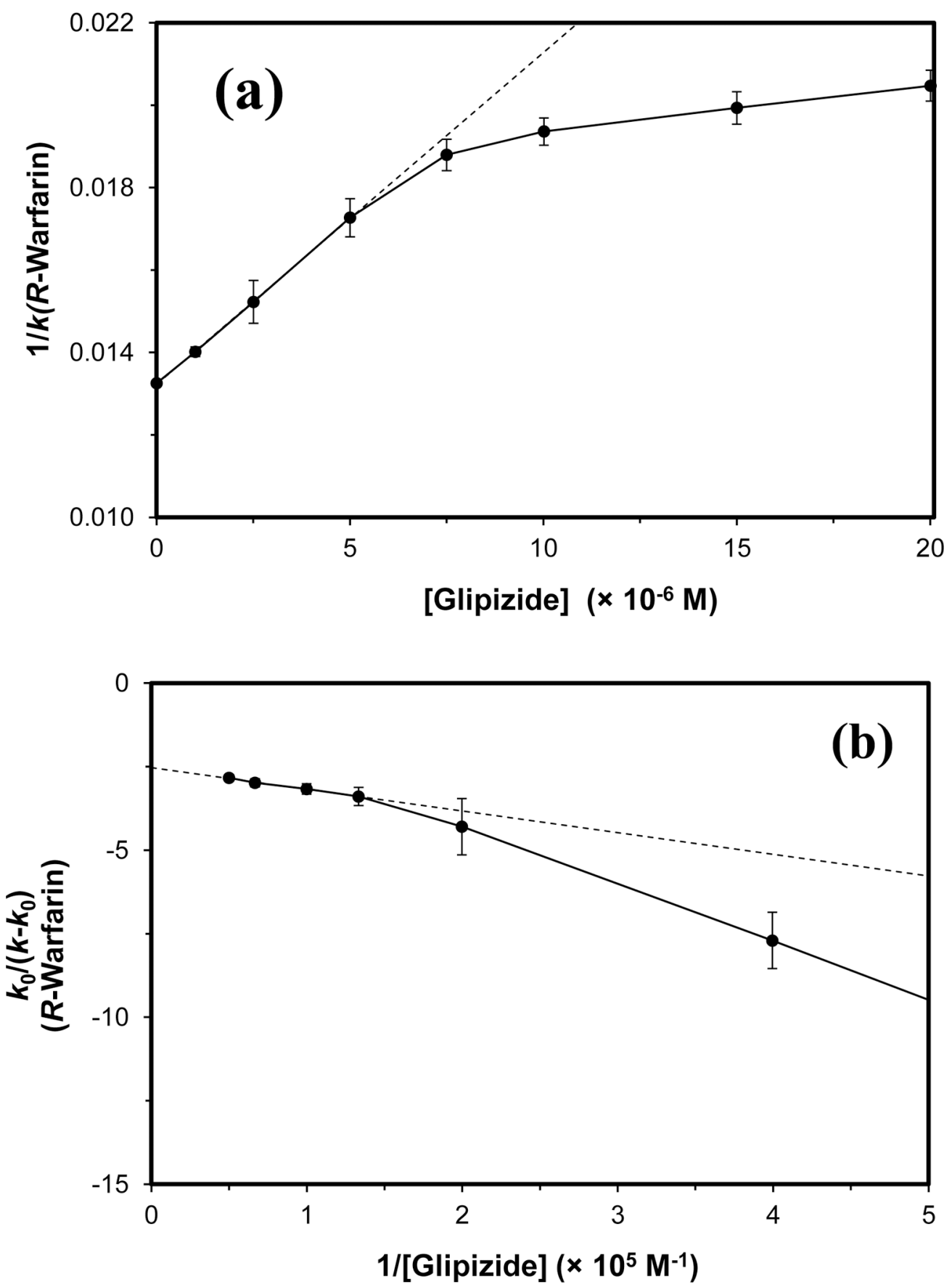

Figure 5.

Plots prepared according to (a) Eq. (6) and (b) Eq. (7) for zonal elution competition studies using glipizide as a competing agent and $R$-warfarin as an injected probe for Sudlow site I. These results were obtained on a $2.0 \mathrm{~cm} \times 2.1 \mathrm{~mm}$ i.d. column containing normal HSA. Other experimental conditions are given in the text. The error bars represent a range of \pm 1 S.D. Each point is the average of four values with relative standard deviations that ranged from (a) $\pm 0.3-3.4 \%$ or (b) $\pm 1.7-2.0 \%$. The equation for the best-fit dashed line in (a) is $y=$ $\left[8.0( \pm 0.1) \times 10^{2}\right] x+\left[1.3( \pm 0.1) \times 10^{5}\right]$, with a correlation coefficient of 0.9999 (over the 
four points on the left side of this plot). The equation for the best-fit dashed line in (b) is $y=$ $\left[-6.5( \pm 0.3) \times 10^{-6}\right] x+[-2.5( \pm 0.1)]$, with a correlation coefficient of 0.9978 (over the four points on the left side of this plot). 

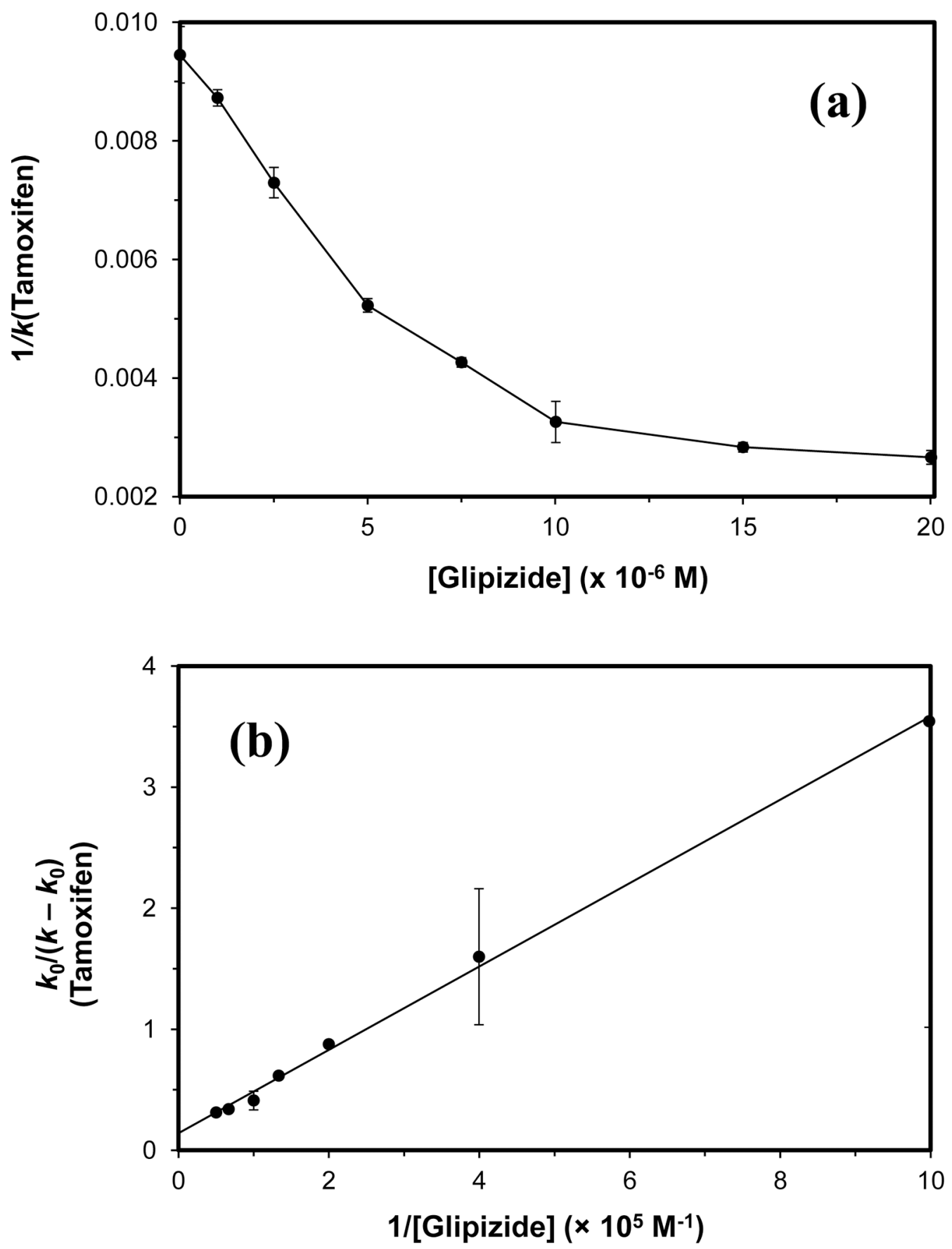

Figure 6.

Plots prepared according to (a) Eq. (6) or (b) Eq. (7) for zonal elution competition studies using glipizide as a competing agent and tamoxifen as an injected probe for the tamoxifen site. These results were obtained on a $2.0 \mathrm{~cm} \times 2.1 \mathrm{~mm}$ i.d. column containing normal HSA. Other experimental conditions are given in the text. The error bars represent a range of \pm 1 S.D. Each point is the average of four values with relative standard deviations that ranged $\pm 1.5-10.6 \%$. The equation for the best-fit solid line in (b) is $y=\left[3.4( \pm 0.1) \times 10^{-6}\right] x+[1.4$ $\left.( \pm 0.3) \times 10^{-1}\right]$, with a correlation coefficient of $0.9990(n=7)$. 


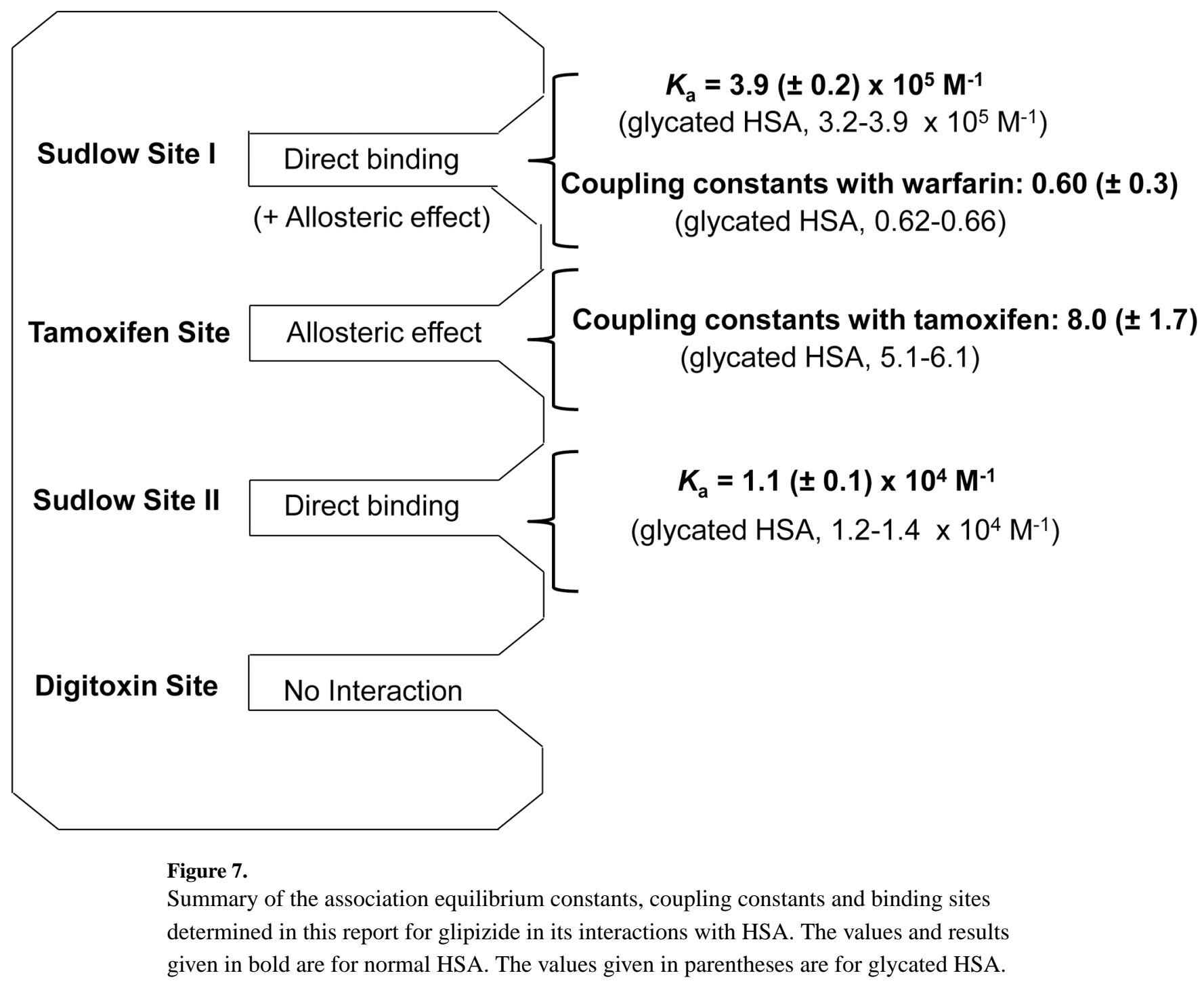


Table 1.

Association equilibrium constants $\left(K_{a}\right)$ and binding capacities $\left(m_{L}\right)$ determined for glipizide with normal HSA or glycated HSA when using a two-site binding model ${ }^{a}$

\begin{tabular}{lcccc}
\hline Type of HSA & $\boldsymbol{K}_{\boldsymbol{a} \mathbf{1}}\left(\mathbf{M}^{\mathbf{- 1} \times \mathbf{1 0}} \mathbf{5}^{\mathbf{5}}\right)$ & $\boldsymbol{m}_{\boldsymbol{L 1} \mathbf{1}}\left(\mathbf{m o l} \times \mathbf{1 0} \mathbf{0}^{\mathbf{- 9}}\right)$ & $\boldsymbol{K}_{\boldsymbol{a} \mathbf{2}}\left(\mathbf{M}^{\left.\mathbf{- 1} \times \mathbf{1 0} \mathbf{0}^{\mathbf{4}}\right)}\right.$ & $\boldsymbol{m}_{\boldsymbol{L} \mathbf{2}}\left(\mathbf{m o l} \times \mathbf{1 0}^{\mathbf{- 8}}\right)$ \\
\hline Normal HSA & $2.4( \pm 0.8)$ & $8.9( \pm 3.0)$ & $1.7( \pm 0.4)$ & $6.0( \pm 0.3)$ \\
gHSA1 & $2.8( \pm 1.1)$ & $9.7( \pm 3.8)$ & $2.5( \pm 0.5)$ & $6.3( \pm 0.2)$ \\
gHSA2 & $6.0( \pm 2.2)$ & $4.2( \pm 1.2)$ & $3.7( \pm 0.2)$ & $5.9( \pm 0.1)$ \\
\hline
\end{tabular}

${ }^{a}$ These results were measured at $37^{\circ} \mathrm{C}$ in the presence of $\mathrm{pH} 7.4,0.067 \mathrm{M}$ potassium phosphate buffer. The values in parentheses represent a range of \pm 1 S.D., as based on error propagation and the precisions of the best-fit slopes and intercepts when using Eq. (3) $(n=12)$. The glycation levels for gHSA1 and gHSA2 were $1.39( \pm 0.28)$ and $3.20( \pm 0.13) \mathrm{mol}$ hexose/mol HSA, respectively. 


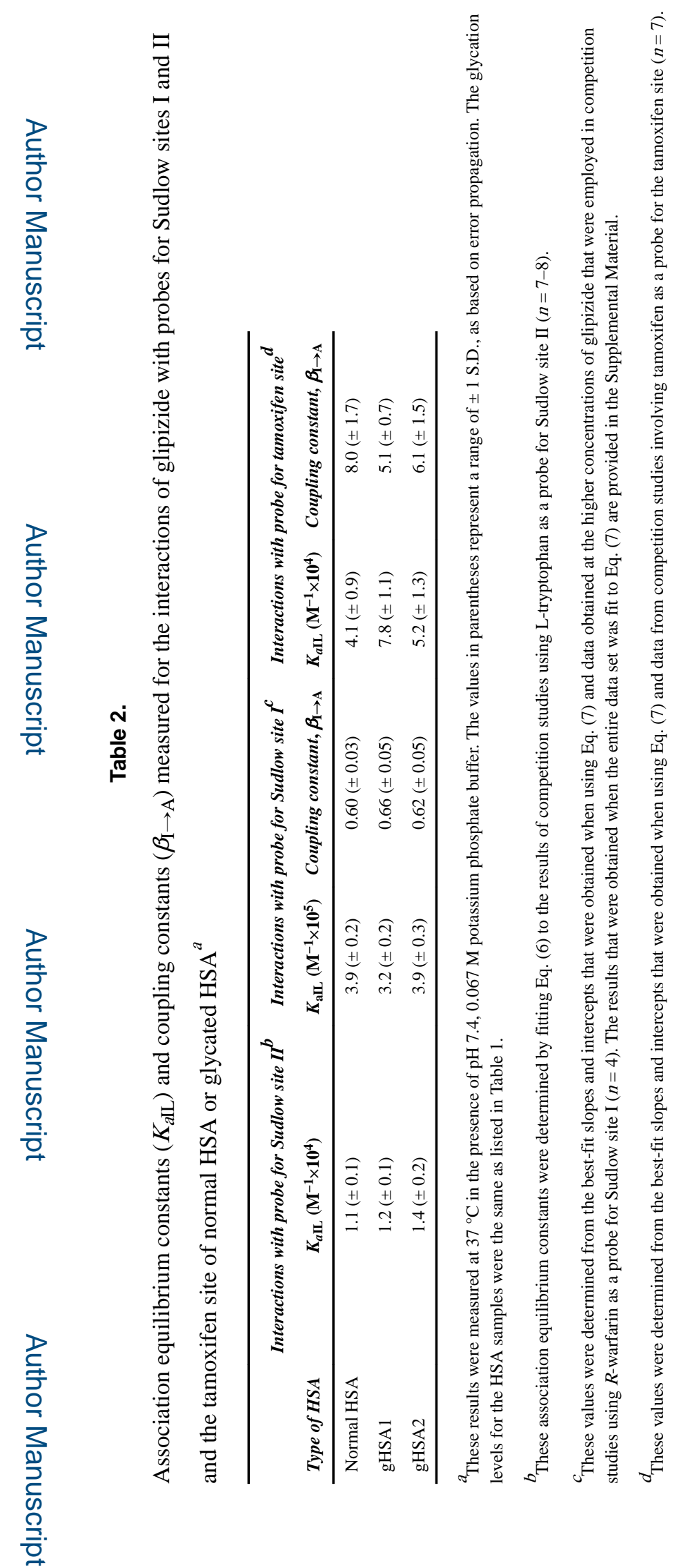

Anal Bioanal Chem. Author manuscript; available in PMC 2019 February 02. 